\title{
Lebensaufgabe Sozialmedizin - Zum 80. Geburtstag von Johannes G. Gostomzyk
}

Autoren

G. v. Mittelstaedt, T. Gaertner, M. Wildner
Bibliografie

DOI http://dx.doi.org/ $10.1055 / \mathrm{s}-0042-101440$

Gesundheitswesen 2016;

78: 69-70

(c) Georg Thieme Verlag KG

Stuttgart $\cdot$ New York

ISSN 0941-3790

Korrespondenzadresse

Dr. med. Gert von

Mittelstaedt

Präsident der DGSMP

Karl-Traub-Straße 18

68199 Mannheim

gertvm@gmx.de
„Die Aufgabe der Sozialmedizin ist es, die im Sektor Medizin einerseits, Gesellschaft anderseits herrschenden Kräfte zu beschreiben und Bilanzierungen unter Aufzeigung aller Alternativen und Konsequenzen zu planen“. Als Summa eines langen Forscherlebens und späten Gelehrtendaseins akzentuiert dieses Diktum Hans Schaefers die Notwendigkeiten, die zur Entstehung des neuen und eigenständigen Fachgebiets in der Medizin führten. Dem wissenschaftlichen Erbe des Nestors der bundesrepublikanischen Sozialmedizin verbunden bildete für Johannes Gostomzyk dieses Paradigma einen Ausgangspunkt des eigenen Engagements zur Konsolidierung und Weiterentwicklung des neu konzipierten Fachs. In ihren Anfangsjahren grenzte sich Sozialmedizin als epidemiologische Methoden anwendende Verbundwissenschaft von Medizin und Soziologie mit Blick auf die Bevölkerungsgruppen und das System der sozialen Sicherung - wenn auch nicht als etwas Alternatives, sondern eher Komplementäres - zunächst klar von der klinischen Individualmedizin $\mathrm{ab}$.

Hier blieb Johannes Gostomzyk nicht stehen; er nahm - diese entfaltend - zentrale Gedanken seines Vorgängers im Amt des Präsidenten der Deutschen Gesellschaft für Sozialmedizin und Prävention (DGSMP), Herbert Viefhues, auf und entwickelte sie weiter. Sozialmedizin war diesem zufolge nämlich nicht nur der Funktionalität des Sozialsystems verschrieben. Eine „verstehende Sozialmedizin“ sollte quasi als „soziale Hilfe“ auch dem Individuum in seiner Lebenswelt nicht zuletzt auch in Form der Prävention - zu Gute kommen: der Sozialmediziner, der als Spezialist eingeht auf den einzelnen Patienten, „auf seine Biografie, seine Familie, seine Arbeitswelt, seine Freunde und Feinde und last but not least, seine Krankheit“. Grundgelegt wurde hiermit der interdisziplinäre Ansatz der Sozialmedizin und damit auch der DGSMP. Als wissenschaftliche Fachgesellschaft fördert sie Forschung, Lehre und Praxis in Sozialmedizin sowie Prävention und führt Vertreterinnen und Vertreter unterschiedlicher Disziplinen zusammen.

Vor dem Hintergrund der Bedeutung von Gesellschaft und Medizin einschließlich Gesundheitsförderung und Prävention als integratives Anliegen einer modernen und belastbaren Sozialmedizin sowie in der Auseinandersetzung mit dem Modell der Salutogenese und den Gedanken Hans Schaefers dazu entwickelte Johannes Gostomzyk in Analogie zu den vielfältigen Krankheiten, die ein Mensch erleiden kann, sein Konzept der vielen Gesundheiten. Diese sind „Aus- druck individueller Reaktionen auf ständig wechselnde Belastungen“ und „die Befindlichkeit des Individuums auf dem Kontinuum zwischen den Polen Gesundheit und Krankheit erscheint durch individuelles und gesellschaftliches Wirken optimierbar“.

Johannes Gostomzyk hat seine Lebensaufgabe einer so umfassend verstandenen Sozialmedizin bestens gerüstet und mit Freude gemeistert: $\mathrm{Er}$ war solide aus- und exzellent weitergebildet, wissenschaftlich qualifiziert, beruflich engagiert und - auch ehrenamtlich - in maßgeblichen Leitungsfunktionen, so auch als Schriftleiter eben des „Gesundheitswesens“, unermüdlich aktiv und stets vielseitig interessiert, Biografien studierend, Kraft suchend - auch in der Philosophie. Ihm wurden für all seine außerordentlichen Verdienste im Jahr 2005 das Bundesverdienstkreuz am Bande und für seine herausragenden Verdienste um die Sozialmedizin im Jahr 2007 die Salomon-Neumann-Medaille der DGSMP verliehen; besonders hervorzuheben ist die Ehrung mit dem Bayerischen Verdienstorden im Jahre 2010. So war Johannes Gostomzyk als Arzt sicher ein Steuermann - „kybernetes“ (Hippokrates), als lehrende, immer auch lernende Integrationsfigur aber vor allem - was seine Ehrungen belegen - in hohem Maße „moderator“ (Isidor von Sevilla), somit ein Mediziner, „der Maß nimmt, Maß hält und dann auch Maßstäbe setzt" - ein Mentor auch, getreu der Schaeferschen Erfahrung, dass Glück nicht einfach Erfolgen entspringe, sondern von den dem „Dienen“ erwachsenen „Verdiensten“ genährt werde.

Mit seinem sozialmedizinischen Wirken hat sich im Laufe seines Lebens eine doppelte Kompetenz herausgebildet, welche auch allgemein kennzeichnend für die Sozialmedizin ist: zum einen die gelebte soziale Verantwortung im ärztlichen Handeln, zum anderen angesichts gesellschaftlicher Defizite die Sorge um eine in ihrem Kern der Humanität und dem sozialen Ausgleich verpflichteten Medizin. Dies betrifft nicht nur praktische Aspekte, sondern bezogen auf die wissenschaftliche Dimension der Sozialmedizin auch deren Reflexion und Theoriebildung im Dienst gelingender und der sozialen Medizin zugewandter, individueller und gesellschaftlicher Interventionen.

Zahlreiche der Sozialmedizin verbundene Praktiker und Wissenschaftler erhielten von Johannes Gostomzyk wegweisende Impulse. Von seiner beachtlichen Wirkkraft wollen die zu diesem Schwerpunktheft eingeladenen Beiträge einen Eindruck vermitteln. So geht einführend Klaus 
Arntz der Frage nach, in wieweit eine moralische Pflicht zur Gesundheit formuliert und ob die Verweigerung gesundheitsbezogenen präventiven Verhaltens zu Recht als unmoralisch bezeichnet werden darf. Martina Enke gibt eine Zusammenschau über die gesundheitsbezogene Prävention der Landeszentrale für Gesundheit in Bayern e.V. - deren 1. Vorsitzender war Gostomzyk von 1997-2013, seitdem Ehrenvorsitzender - mit dem Anspruch der Bedarfsorientierung und des Pragmatismus. Christa Meisinger berichtet den Werdegang der epidemiologischen Forschung im Raum Augsburg vom MONIKA-Projekt über KORA bis zur Studienregion der nationalen Kohorte und analysiert den praktischen Nutzen von Bevölkerungsstudien für eine Region. Holger Pfaff und Timo-Kolja Pförtner diskutieren den wissenschaftlich jüngeren, durchaus aufstrebenden Zweig der Versorgungsforschung und dessen Beitrag zur Chancengerechtigkeit in der gesundheitlichen Versorgung. Elisabeth Simoes wirbt für eine Vermittlung sozialmedizinischer Inhalte im Rahmen der klinisch-fachärztlichen Weiterbildung und folgt dabei der Kausalkette ausgehend von der epidemiologischen Assoziation über feststellbare Krankheitsursachen bis hin zur Sozialmedizin in der akut-klinischen Versorgung der Universitäts-Frauenklinik Tübingen. Ulrich Storr, Anton Euba und Elisabeth Paschos greifen das große und für die Bevölkerungsmedizin wichtige Thema einer nachhaltigen Zahngesundheit am Thema der Motivation zur Prävention im Kindesalter auf. Gerhard Trabert thematisiert die medizinische Versorgung obdachloser Menschen; richtet den Blick auf die Fragestellung, in wieweit es sich hier aus menschrechtlicher Sicht um eine soziale Pflicht handelt und ob dieser Ansatz eine nachhaltige gesellschaftliche Lösung gegenüber der noch immer oft zu beobachtenden sozialen Exklusion darstellen kann. Manfred Wildner, Wolfgang Hofmann und JensUwe Niehoff reflektieren die Entwicklungslinien der Begriffe Öffentliche Gesundheit und Sozialmedizin vor dem historisch empfundenen Spannungsfeld der Wiedervereinigung der beiden deutschen Staaten mit ihren unterschiedlichen Traditionslinien und den Besonderheiten der Multidisziplin Public Health. Abschließend nehmen Gert von Mittelstaedt und Thomas Gaertner aus der Perspektive der Fachgesellschaft sowie der praktischen Sozialmedizin Stellung zur interdisziplinären Ausrichtung der DGSMP vor dem Hintergrund des versorgungsrelevanten Aufgabenspektrums einer zeitgemäßen Sozialmedizin als integratives Fachgebiet der Leitdisziplin Medizin - ganz in der Traditionslinie von Schaefer und Gostomzyk weitergeführt bis heute. Dem Jubilar wünschen wir alles Gute für die Zukunft sowie mit dem Novalis-Wort „Ein wahrer Forscher wird nicht alt“ ungebrochene Schaffenskraft. Für unsere Leserinnen und Leser erhoffen wir interessante Anregungen für eine anhaltende und fruchtbare Auseinandersetzung mit der „Lebensaufgabe Sozialmedizin“! 